\title{
FUNDAMENTAL REGIONS FOR THE SIMPLE GROUP OF ORDER 60 IN $S_{4}$
}

\author{
R. R. STOLL
}

Introduction. By a finite collineation group of order $g$, a point will be transformed into $g$ points at the most. A set of points which are permuted among themselves by the operators of the group are said to form a conjugate set of points. A fundamental region in the plane for the group is defined as a system of points which contains one and only one point from every conjugate set. Therefore, for a collineation group of order $g$, there are $g$ fundamental regions.

For example, consider the group of order four generated by the transformations $x=y^{\prime}, y=-x^{\prime}$. These transformations consist of the rotations of a point through one, two, three, and four right angles about the origin in the $x y$-plane. Any two perpendicular lines through the origin divide the plane into four parts which are the fundamental regions for the group on the plane. Points on the two lines themselves may be assigned to either of the two adjacent regions, provided that no point and its transform be put in the same fundamental region. This example demonstrates that the solution of such a problem is not unique.

The definition of fundamental regions is readily extended to groups in more than two variables, and in the case of three variables the problem has received some attention. J. W. Young* obtained a solution for cyclic groups, W. I. Miller $\dagger$ for the $G_{168}$, and H. F. Price $\ddagger$ for the $G_{24}$ and the $G_{60}$ when written with real coefficients in every transformation. This paper is devoted to the determination of fundamental regions for the $G_{60}$ when written with complex coefficients in its transformations. The method used is similar to that used by Miller for the $G_{168}$.

Determination of fundamental regions. Considered as a collineation group, the generating substitutions for the $G_{60}$ are taken to be $\S$

* J. W. Young, Fundamental regions for cyclic groups of linear fractional transformations on two complex variables, this Bulletin, vol. 17 (1910-1911), pp. 340-344.

$\dagger$ W. I. Miller, Fundamental regions for the simple group of order 168 in $S_{4}$, American Journal of Mathematics, vol. 56 (1934), pp. 316-318.

$\ddagger$ H. F. Price, Fundamental regions for certain finite groups in $S_{4}$, American Journal of Mathematics, vol. 40 (1919), pp. 108-113.

$\S$ H. F. Blichfeldt, Finite Collineation Groups, University of Chicago Press, 1917, chap. 5 , p. 114 . 


$$
\begin{aligned}
& S: x_{0}=x_{0}^{\prime}, \quad x_{1}=\omega^{4} x_{1}^{\prime}, \quad x_{2}=\omega x_{2}^{\prime}, \\
& T: \begin{array}{l}
x_{0}=1 / 5^{1 / 2}\left(x_{0}^{\prime}+x_{1}^{\prime}+x_{2}^{\prime}\right), \quad x_{1}=1 / 5^{1 / 2}\left(2 x_{0}^{\prime}+b x_{1}^{\prime}+a x_{2}^{\prime}\right), \\
x_{2}=1 / 5^{1 / 2}\left(2 x_{0}^{\prime}+a x_{1}^{\prime}+b x_{2}^{\prime}\right),
\end{array}
\end{aligned}
$$

where $\omega$ is the principal fifth root of unity, $a=\omega+\omega^{4}, b=\omega^{2}+\omega^{3}$.

Let the homogeneous complex variables $x_{0}, x_{1}, x_{2}$ be represented by real points in the $S_{4}$ of $x, y, u, v$ where $x_{0} / x_{2}=x+i u, x_{1} / x_{2}=y+i v$. It is understood that when the variables are transformed by an operator of the group, the conjugate imaginary variables $\bar{x}_{0}, \bar{x}_{1}, \bar{x}_{2}$ are transformed by the conjugate imaginary operator.

Consider the six positive forms $A_{i}=f_{i} \bar{f}_{i},(i=0,1, \cdots, 5)$, where $\bar{f}_{i}$ is the conjugate imaginary of $f_{i}$ and $f_{0}=5^{1 / 2} x_{0}, f_{i}=x_{0}+\omega^{1-i} x_{1}+\omega^{i-1} x_{2}$, $(i=1,2, \cdots, 5)$. These forms are permuted by $S$ and $T$ :

$$
S: A_{1} A_{2} A_{3} A_{4} A_{5} \cdot, \quad T: A_{0} A_{1} \cdot A_{2} A_{5} \cdot .
$$

When writing the $G_{60}$ as a permutation group of degree six, we may choose as the generating substitutions $S=\left(A_{1} A_{2} A_{3} A_{4} A_{5}\right)$ and $T=\left(A_{0} A_{1}\right)\left(A_{2} A_{5}\right)$.

Forming the differences of the forms $A_{0}, \cdots, A_{5}$ gives 15 indefinite forms $B_{i j},(i=1,2,3 ; j=0,1, \cdots, 4)$, where $B_{10}=A_{0}-A_{1}$, $B_{20}=A_{1}-A_{2}, B_{30}=A_{1}-A_{3}$, and the others are selected so that $S$ permutes them in the order $B_{i, j}, B_{i, j+1}$. For example,

$B_{10}=4 x_{0} \bar{x}_{0}-x_{0} \bar{x}_{1}-x_{0} \bar{x}_{2}-x_{1} \bar{x}_{0}-x_{1} \bar{x}_{1}-x_{1} \bar{x}_{2}-x_{2} \bar{x}_{0}-x_{2} \bar{x}_{1}-x_{2} \bar{x}_{2}$.

These 15 forms are used to determine the fundamental regions for the $G_{60}$. In order to change from the homogeneous complex variables $x_{0}, x_{1}, x_{2}$ and their conjugate imaginaries to the nonhomogeneous real variables $x, y, u, v$ in one of the forms, it is necessary to divide the form by $x_{2} \bar{x}_{2}$. If we perform the division on a form and transform the result by an operator that does not leave $x_{2}$ fixed, the denominator will be positive and may be replaced by $x_{2} \bar{x}_{2}$ without altering the sign of the form. For certain values of the variables one or more of the denominators will vanish; however, they cannot all vanish at once, since the variables $x_{0}, x_{1}, x_{2}$ represent homogeneous coordinates of points in a plane.

Dividing each form by $x_{2} \bar{x}_{2}$ and replacing the fractions by their equivalents in terms of $x, y, u, v$ gives, if each result is equated to zero, 15 hypersurfaces in $S_{4}$. If we exclude points on one or more of the hypersurfaces, a point of $S_{4}$ will make each of these forms either positive or negative. There will be at most 6 ! possible arrangements of sign as these 15 forms are the differences of six positive forms. Later it is shown that all of the $6 !$ arrangements are present. An arrange- 
ment of signs is most conveniently given by writing the order of magnitude of the six forms $A_{0}, \cdots, A_{5}$; and if $A_{0}>A_{1}>A_{2}>A_{3}>A_{4}>A_{5}$, we shall denote this by $A_{0} A_{1} A_{2} A_{3} A_{4} A_{5}$. This means $B_{10}>0, B_{20}>0$, and so on. If we assume, for the present, that every value system (that is, every arrangement of magnitude) of $A_{0}, \cdots, A_{5}$ is possible, it follows that $S_{4}$ is divided into 6 ! regions by the 15 forms, and no operator of the group except the identity transforms a point of one region into a point of the same region, since no operator on $A_{0}, \cdots, A_{5}$, except the identity, leaves a given arrangement of letters unchanged. Thus each region has 60 conjugates under the group; and since $6 !=60 \times 12$, there are 12 such sets of conjugates. To obtain a fundamental region we select one region from each of the 12 sets.

Let us formulate a scheme to determine to which one of the 12 sets, which we shall denote by $S_{i j},(i=1,2 ; j=1,2, \cdots, 6)$, a given value system of $A_{0}, \cdots, A_{5}$ belongs. The following rules serve to classify the different value systems:

(a) If the first letter is not $A_{0}$ (say $A_{k}$ ), transform the value system by a suitable operator of $G_{60}$ so that $A_{k}$ is replaced by $A_{0}$. The operator $S^{-i} T S^{i}$, which represents

$$
\begin{aligned}
\left(A_{0} A_{1}\right)\left(A_{2} A_{5}\right), \quad\left(A_{0} A_{2}\right)\left(A_{1} A_{3}\right), & \left(A_{0} A_{3}\right)\left(A_{2} A_{4},\right) \\
& \left(A_{0} A_{4}\right)\left(A_{3} A_{5}\right), \quad\left(A_{0} A_{5}\right)\left(A_{1} A_{4}\right)
\end{aligned}
$$

for $i=0,1,2,3,4$, serves this purpose.

(b) If the second letter is not $A_{1}$, transform by an operator of the $G_{60}$ that omits $A_{0}$ and replaces the second letter by $A_{1}$. A power of $S=\left(A_{1} A_{2} A_{3} A_{4} A_{5}\right)$ will accomplish this.

(c) If the third letter is neither $A_{2}$ nor $A_{3}$, transform by $S^{2} T S\left(S^{2} T\right)^{2}=\left(A_{2} A_{5}\right)\left(A_{3} A_{4}\right)$; then our value system will have one of the two forms $A_{0} A_{1} A_{2} x x x, A_{1} A_{2} A_{3} x x x$, where the $x$ 's denote any of the remaining letters. The first subscript is determined as follows: $S_{1 j}$ contains $A_{0} A_{1} A_{2} x x x ; S_{2 j}$ contains $A_{0} A_{1} A_{3} x x x$.

(d) The second subscript is determined by the arrangement of the remaining three letters in $S_{i j}$. The symbol $A_{p}$ denotes either $A_{2}$ or $A_{3}$ :

\begin{tabular}{|l|cccccc|}
\hline Order of $A_{p}, A_{4}, A_{5}$ & $A_{p} A_{4} A_{5}$ & $A_{p} A_{5} A_{4}$ & $A_{4} A_{p} A_{5}$ & $A_{5} A_{p} A_{4}$ & $A_{4} A_{5} A_{p}$ & $A_{5} A_{4} A_{p}$ \\
\hline Second subscript & 1 & 2 & 3 & 4 & 5 & 6 \\
\hline
\end{tabular}

Example. $A_{1} A_{3} A_{5} A_{0} A_{2} A_{4}$ is transformed into $A_{0} A_{1} A_{2} A_{3} A_{4} A_{5}$ by the above rules; hence it belongs to $S_{11}$.

It is interesting to study the intersections of the fundamental regions with certain planes in $S_{4}$; in addition this enables us to prove 
the existence of the 12 sets of regions $S_{i j}$ by exhibiting points in each set. We shall mention only two planes. (i) Consider the plane $x=0$, $y=0$. The hypersurfaces each intersect this plane in a distinct curve. Included in the intersections are 13 hyperbolas and two straight lines. Upon computing the value systems for the regions intersected by this plane we find that regions from each of the 12 sets $S_{i j}$ are represented. (ii) Consider the plane $u=0, v=0$. The forms $B_{22}$ and $B_{34}$ vanish for every point of this plane; it is therefore part of a boundary region. Each region of the plane is on the boundary of four regions in $S_{4}$.

The existence of the 12 sets of regions $S_{i j}$ with 60 regions to a set means that it is possible to obtain points whose coordinates give the six forms $A_{0}, \cdots, A_{5}$ any preassigned order of magnitude, excluding cases in which two or more of the forms are equal. As stated before, a fundamental region is obtained by selecting one value system for $A_{0}, \cdots, A_{5}$ from each of the 12 sets; all points whose coordinates arrange these forms in any one of the 12 value systems thus selected belongs to the same fundamental region. Each fundamental region has 60 conjugates under the group.

As an example of a fundamental region we take the 12 value systems into which all others are transformed by the rules stated above. These are the value systems for which

$$
A_{0}>A_{1}>A_{2}, \quad A_{1}>A_{3}, \quad A_{1}>A_{4}, \quad A_{1}>A_{5} ;
$$

and, furthermore, the quantity next in order of magnitude to $A_{1}$ is either $A_{2}$ or $A_{3}$. To complete the determination of the fundamental region given above we assign to it suitable points on the hypersurfaces $B_{i j}=0$. We start with the 12 value systems in which the forms $A_{0}, \cdots, A_{5}$ are all distinct and set two or more of the forms equal in all possible ways. After discarding duplicates, we arrange these value systems into sets of conjugates under the group and from each conjugate set select one value system. The value systems thus obtained, together with the 12 in which no two forms are equal, give a complete determination of the fundamental region.

Rensselaer Polytechnic Institute 